\title{
Positive solutions for a sixth-order boundary value problem with four parameters
}

Ravi P Agarwall ${ }^{1 *}$, B Kovacs $^{2}$ and D O'Regan ${ }^{3}$

${ }^{\text {"Correspondence: }}$

Agarwal@tamuk.edu

${ }^{1}$ Department of Mathematics, Texas A\&M University-Kingsville, 700

University Blvd., Kingsville,

78363-8202, USA

Full list of author information is

available at the end of the article

\begin{abstract}
This paper investigates the existence and multiplicity of positive solutions of a sixth-order differential system with four variable parameters using a monotone iterative technique and an operator spectral theorem.
\end{abstract}

MSC: $34 \mathrm{~B} 15 ; 34 \mathrm{~B} 18$

Keywords: positive solutions; variable parameters; fixed point theorem; operator spectral theorem

\section{Introduction}

It is well known that boundary value problems for ordinary differential equations can be used to describe a large number of physical, biological and chemical phenomena. In recent years, boundary value problems for sixth-order ordinary differential equations, which arise naturally, for example, in sandwich beam deflection under transverse shear have been studied extensively, see [1-4] and the references therein. The deformation of the equilibrium state of an elastic circular ring segment with its two ends simply supported can be described by a boundary value problem involving a sixth-order ordinary differential equation

$$
\begin{aligned}
& u^{(6)}+2 u^{(4)}+u^{\prime \prime}=f(t, u), \quad 0<t<1, \\
& u(0)=u(1)=u^{\prime \prime}(0)=u^{\prime \prime}(1)=u^{(4)}(0)=u^{(4)}(1)=0 .
\end{aligned}
$$

Liu and Li [5] studied the existence and nonexistence of positive solutions of the nonlinear fourth-order beam equation

$$
\begin{aligned}
& u^{(4)}(t)+\beta u^{\prime \prime}(t)-\alpha u(t)=\lambda f(t, u(t)), \quad 0<t<1, \\
& u(0)=u(1)=u^{\prime \prime}(0)=u^{\prime \prime}(1)=0 .
\end{aligned}
$$

They showed that there exists a $\lambda^{\star}>0$ such that the above boundary value problem has at least two, one, and no positive solutions for $0<\lambda<\lambda^{\star}, \lambda=\lambda^{\star}$ and $\lambda>\lambda^{\star}$, respectively.

In this paper, we discuss the existence of positive solutions for the sixth-order boundary value problem

$$
\begin{aligned}
& -u^{(6)}+A(t) u^{(4)}+B(t) u^{\prime \prime}+C(t) u=(D(t)+u) \varphi+\lambda f(t, u), \quad 0<t<1, \\
& -\varphi^{\prime \prime}+\varkappa \varphi=\mu u, \quad 0<t<1,
\end{aligned}
$$




$$
\begin{aligned}
& u(0)=u(1)=u^{\prime \prime}(0)=u^{\prime \prime}(1)=u^{(4)}(0)=u^{(4)}(1)=0, \\
& \varphi(0)=\varphi(1)=0 .
\end{aligned}
$$

For this, we shall assume the following conditions throughout

(H1) $f(t, u):[0,1] \times[0, \infty) \longrightarrow[0, \infty)$ is continuous;

(H2) $a, b, c \in R, a=\lambda_{1}+\lambda_{2}+\lambda_{3}>-\pi^{2}, b=-\lambda_{1} \lambda_{2}-\lambda_{2} \lambda_{3}-\lambda_{1} \lambda_{3}>0, c=\lambda_{1} \lambda_{2} \lambda_{3}<0$

where $\lambda_{1} \geq 0 \geq \lambda_{2} \geq-\pi^{2}, 0 \leq \lambda_{3}<-\lambda_{2}$ and $\pi^{6}+a \pi^{4}-b \pi^{2}+c>0$, and

$A, B, C, D \in C[0,1]$ with $a=\sup _{t \in[0,1]} A(t), b=\inf _{t \in[0,1]} B(t)$ and $c=\sup _{t \in[0,1]} C(t)$.

Let $K=\max _{0 \leq t \leq 1}[-A(t)+B(t)-C(t)-(-a+b-c)]$ and $\Gamma=\pi^{6}+a \pi^{4}-b \pi^{2}+c$.

Assumption (H2) involves a three-parameter nonresonance condition.

More recently Li [6] studied the existence and multiplicity of positive solutions for a sixth-order boundary value problem with three variable coefficients. The main difference between our work and [6] is that we consider boundary value problem not only with three variable coefficients, but also with two positive parameters $\lambda$ and $\mu$, and the existence of the positive solution depends on these parameters. In this paper, we shall apply the monotone iterative technique [7] to boundary value problem (3) and then obtain several new existence and multiplicity results. In the special case, in [8] by using the fixed point theorem and the operator spectral theorem, we establish a theorem on the existence of positive solutions for the sixth-order boundary value problem (3) with $\lambda=1$.

\section{Preliminaries}

Let $Y=C[0,1]$ and $Y_{+}=\{u \in Y: u(t) \geq 0, t \in[0,1]\}$. It is well known that $Y$ is a Banach space equipped with the norm $\|u\|_{0}=\sup _{t \in[0,1]}|u(t)|$. Set $X=\left\{u \in C^{4}[0,1]: u(0)=u(1)=\right.$ $\left.u^{\prime \prime}(0)=u^{\prime \prime}(1)=0\right\}$. For given $\chi \geq 0$ and $v \geq 0$, we denote the norm $\|\cdot\|_{\chi, v}$ by

$$
\|\cdot\|_{\chi, v}=\sup _{t \in[0,1]}\left\{\left|u^{(4)}(t)\right|+\chi\left|u^{\prime \prime}(t)\right|+v|u(t)|\right\}, \quad u \in X
$$

We also need the space $X$, equipped with the norm

$$
\|u\|_{2}=\max \left\{\|u\|_{0},\left\|u^{\prime \prime}\right\|_{0},\left\|u^{(4)}\right\|_{0}\right\} .
$$

In [8], it is shown that $X$ is complete with the norm $\|\cdot\|_{\chi, v}$ and $\|u\|_{2}$, and moreover $\forall u \in X$, $\|u\|_{0} \leq\left\|u^{\prime \prime}\right\|_{0} \leq\left\|u^{(4)}\right\|_{0}$.

For $h \in Y$, consider the linear boundary value problem

$$
\begin{aligned}
& -u^{(6)}+a u^{(4)}+b u^{\prime \prime}+c u=h(t), \quad 0<t<1, \\
& u(0)=u(1)=u^{\prime \prime}(0)=u^{\prime \prime}(1)=u^{(4)}(0)=u^{(4)}(1)=0,
\end{aligned}
$$

where $a, b, c$ satisfy the assumption

$$
\pi^{6}+a \pi^{4}-b \pi^{2}+c>0
$$

and let $\Gamma=\pi^{6}+a \pi^{4}-b \pi^{2}+c$. Inequality (5) follows immediately from the fact that $\Gamma=$ $\pi^{6}+a \pi^{4}-b \pi^{2}+c$ is the first eigenvalue of the problem $-u^{(6)}+a u^{(4)}+b u^{\prime \prime}+c u=\lambda u, u(0)=$ $u(1)=u^{\prime \prime}(0)=u^{\prime \prime}(1)=u^{(4)}(0)=u^{(4)}(1)=0$, and $\phi_{1}(t)=\sin \pi t$ is the first eigenfunction, i.e. 
$\Gamma>0$. Since the line $l_{1}=\left\{(a, b, c): \pi^{6}+a \pi^{4}-b \pi^{2}+c=0\right\}$ is the first eigenvalue line of the three-parameter boundary value problem $-u^{(6)}+a u^{(4)}+b u^{\prime \prime}+c u=0, u(0)=u(1)=$ $u^{\prime \prime}(0)=u^{\prime \prime}(1)=u^{(4)}(0)=u^{(4)}(1)=0$, if $(a, b, c)$ lies in $l_{1}$, then by the Fredholm alternative, the existence of a solution of the boundary value problem (4) cannot be guaranteed.

Let $P(\lambda)=\lambda^{2}+\beta \lambda-\alpha$, where $\beta<2 \pi^{2}, \alpha \geq 0$. It is easy to see that the equation $P(\lambda)=0$ has two real roots $\lambda_{1}, \lambda_{2}=\frac{-\beta \pm \sqrt{\beta^{2}+4 \alpha}}{2}$ with $\lambda_{1} \geq 0 \geq \lambda_{2}>-\pi^{2}$. Let $\lambda_{3}$ be a number such that $0 \leq \lambda_{3}<-\lambda_{2}$. In this case, (4) satisfies the decomposition form

$$
-u^{(6)}+a u^{(4)}+b u^{\prime \prime}+c u=\left(-\frac{d^{2}}{d t^{2}}+\lambda_{1}\right)\left(-\frac{d^{2}}{d t^{2}}+\lambda_{2}\right)\left(-\frac{d^{2}}{d t^{2}}+\lambda_{3}\right) u, \quad 0<t<1 .
$$

Suppose that $G_{i}(t, s)(i=1,2,3)$ is the Green's function associated with

$$
-u^{\prime \prime}+\lambda_{i} u=0, \quad u(0)=u(1)=0 .
$$

We need the following lemmas.

Lemma $1[5,9]$ Let $\omega_{i}=\sqrt{\left|\lambda_{i}\right|}$, then $G_{i}(t, s)(i=1,2,3)$ can be expressed as

(i) when $\lambda_{i}>0$,

$$
G_{i}(t, s)=\left\{\begin{array}{l}
\frac{\sinh \omega_{i} t \sinh \omega_{i}(1-s)}{\omega_{i} \sinh \omega_{i}}, 0 \leq t \leq s \leq 1 \\
\frac{\sinh \omega_{i} s \sinh \omega_{i}(1-t)}{\omega_{i} \sinh \omega_{i}}, 0 \leq s \leq t \leq 1
\end{array}\right\}
$$

(ii) when $\lambda_{i}=0$,

$$
G_{i}(t, s)=\left\{\begin{array}{l}
t(1-s), 0 \leq t \leq s \leq 1 \\
s(1-t), 0 \leq s \leq t \leq 1
\end{array}\right\}
$$

(iii) when $-\pi^{2}<\lambda_{i}<0$,

$$
G_{i}(t, s)=\left\{\begin{array}{l}
\frac{\sin \omega_{i} t \sin \omega_{i}(1-s)}{\omega_{i} \sin \omega_{i}}, 0 \leq t \leq s \leq 1 \\
\frac{\sin \omega_{i} \sin \omega_{i}(1-t)}{\omega_{i} \sin \omega_{i}}, 0 \leq s \leq t \leq 1
\end{array}\right\} .
$$

Lemma $2[5] G_{i}(t, s)(i=1,2,3)$ has the following properties

(i) $G_{i}(t, s)>0, \forall t, s \in(0,1)$;

(ii) $G_{i}(t, s) \leq C_{i} G_{i}(s, s), \forall t, s \in[0,1]$;

(iii) $G_{i}(t, s) \geq \delta_{i} G_{i}(t, t) G_{i}(s, s), \forall t, s \in[0,1]$,

where $C_{i}=1, \delta_{i}=\frac{\omega_{i}}{\sinh \omega_{i}}$, if $\lambda_{i}>0 ; C_{i}=1, \delta_{i}=1$, if $\lambda_{i}=0 ; C_{i}=\frac{1}{\sin \omega_{i}}, \delta_{i}=\omega_{i} \sin \omega_{i}$, if $-\pi^{2}<\lambda_{i}<0$.

In what follows, we let $D_{i}=\max _{t \in[0,1]} \int_{0}^{1} G_{i}(t, s) d s$.

Lemma 3 [10] Let $X$ be a Banach space, $K$ a cone and $\Omega$ a bounded open subset of $X$. Let $\theta \in \Omega$ and $T: K \cap \bar{\Omega} \rightarrow K$ be condensing. Suppose that $T x \neq v x$ for all $x \in K \cap \partial \Omega$ and $v \geq 1$. Then $i(T, K \cap \Omega, K)=1$. 
Lemma 4 [10] Let $X$ be a Banach space, let $K$ be a cone of $X$. Assume that $T: \bar{K}_{r} \rightarrow K$ (here $\left.K_{r}=\{x \in K \mid\|x\|<r\}, r>0\right)$ is a compact map such that Tx $\neq x$ for all $x \in \partial K_{r}$. If $\|x\| \leq\|T x\|$ for $x \in \partial K_{r}$, then $i\left(T, K_{r}, K\right)=0$.

Now, since

$$
\begin{aligned}
-u^{(6)}+a u^{(4)}+b u^{\prime \prime}+c u & =\left(-\frac{d^{2}}{d t^{2}}+\lambda_{1}\right)\left(-\frac{d^{2}}{d t^{2}}+\lambda_{2}\right)\left(-\frac{d^{2}}{d t^{2}}+\lambda_{3}\right) u \\
& =\left(-\frac{d^{2}}{d t^{2}}+\lambda_{2}\right)\left(-\frac{d^{2}}{d t^{2}}+\lambda_{1}\right)\left(-\frac{d^{2}}{d t^{2}}+\lambda_{3}\right) u=h(t),
\end{aligned}
$$

the solution of boundary value problem (4) can be expressed as

$$
u(t)=\int_{0}^{1} \int_{0}^{1} \int_{0}^{1} G_{1}(t, v) G_{2}(\nu, s) G_{3}(s, \tau) h(\tau) d \tau d s d \nu, \quad t \in[0,1] .
$$

Thus, for every given $h \in Y$, the boundary value problem (4) has a unique solution $u \in$ $C^{6}[0,1]$, which is given by $(9)$.

We now define a mapping $T: C[0,1] \rightarrow C[0,1]$ by

$$
(T h)(t)=\int_{0}^{1} \int_{0}^{1} \int_{0}^{1} G_{1}(t, v) G_{2}(v, s) G_{3}(s, \tau) h(\tau) d \tau d s d v, \quad t \in[0,1]
$$

Throughout this article, we shall denote $T h=u$ the unique solution of the linear boundary value problem (4).

Lemma 5 [8] $T: Y \longrightarrow\left(X,\|\cdot\|_{\chi, v}\right)$ is linear completely continuous, where $\chi=\lambda_{1}+\lambda_{3}$, $v=\lambda_{1} \lambda_{3}$ and $\|T\| \leq D_{2}$. Moreover, $\forall h \in Y_{+}$, if $u=T h$, then $u \in X \cap Y_{+}$, and $u^{\prime \prime} \leq 0, u^{(4)} \geq 0$.

We list the following conditions for convenience

(H3) $f(t, u)$ is nondecreasing in $u$ for $t \in[0,1]$;

(H4) $f(t, 0)>\widehat{c}>0$ for all $t \in[0,1]$;

(H5) $f_{\infty}=\lim _{u \rightarrow \infty} \frac{f(t, u)}{u}=\infty$ uniformly for $t \in[0,1]$;

(H6) $f(t, \rho u) \geq \rho^{\alpha} f(t, u)$ for $\rho \in(0,1)$ and $t \in[0,1]$, where $\alpha \in(0,1)$ is independent of $\rho$ and $u$.

Suppose that $G(t, s)$ is the Green's function of the linear boundary value problem

$$
-u^{\prime \prime}+\varkappa u=0, \quad u(0)=u(1)=0 .
$$

Then, the boundary value problem

$$
-\varphi^{\prime \prime}+\varkappa \varphi=\mu u, \quad \varphi(0)=\varphi(1)=0
$$

can be solved by using Green's function, namely,

$$
\varphi(t)=\mu \int_{0}^{1} G(t, s) u(s) d s, \quad 0<t<1
$$


where $\varkappa>-\pi^{2}$. Thus, inserting (12) into the first equation in (3), yields

$$
\begin{aligned}
& -u^{(6)}+A(t) u^{(4)}+B(t) u^{\prime \prime}+C(t) u=\mu(D(t)+u(t)) \int_{0}^{1} G(t, s) u(s) d s+\lambda f(t, u), \\
& u(0)=u(1)=u^{\prime \prime}(0)=u^{\prime \prime}(1)=u^{(4)}(0)=u^{(4)}(1)=0 .
\end{aligned}
$$

Let us consider the boundary value problem

$$
\begin{aligned}
& -u^{(6)}+A(t) u^{(4)}+B(t) u^{\prime \prime}+C(t) u=h(t), \quad 0<t<1, \\
& u(0)=u(1)=u^{\prime \prime}(0)=u^{\prime \prime}(1)=u^{(4)}(0)=u^{(4)}(1)=0 .
\end{aligned}
$$

Now, we consider the existence of a positive solution of (14). The function $u \in C^{6}(0,1) \cap$ $C^{4}[0,1]$ is a positive solution of (14), if $u \geq 0, t \in[0,1]$, and $u \neq 0$.

Let us rewrite equation (13) in the following form

$$
\begin{aligned}
-u^{(6)}+a u^{(4)}+b u^{\prime \prime}+c u= & -(A(t)-a) u^{(4)}-(B(t)-b) u^{\prime \prime}-(C(t)-c) u \\
& +\mu(D(t)+u(t)) \int_{0}^{1} G(t, s) u(s) d s+h(t) .
\end{aligned}
$$

For any $u \in X$, let

$$
G u=-(A(t)-a) u^{(4)}-(B(t)-b) u^{\prime \prime}-(C(t)-c) u+\mu D(t) \int_{0}^{1} G(t, s) u(s) d s .
$$

The operator $G: X \rightarrow Y$ is linear. By Lemmas 2 and 3 in [8], $\forall u \in X, t \in[0,1]$, we have

$$
\begin{aligned}
|(G u)(t)| & \leq[-A(t)+B(t)-C(t)-(-a+b-c)]\|u\|_{2}+\mu C d_{1}\|u\|_{0} \\
& \leq\left(K+\mu C d_{1}\right)\|u\|_{2} \leq\left(K+\mu C d_{1}\right)\|u\|_{\chi, v}
\end{aligned}
$$

where $C=\max _{t \in[0,1]} D(t), K=\max _{t \in[0,1]}[-A(t)+B(t)-C(t)-(-a+b-c)], d_{1}=$ $\max _{t \in[0,1]} \int_{0}^{1} G(t, s) d s, \chi=\lambda_{1}+\lambda_{3} \geq 0, v=\lambda_{1} \lambda_{3} \geq 0$. Hence $\|G u\|_{0} \leq\left(K+\mu C d_{1}\right)\|u\|_{\chi, v}$, and so $\|G\| \leq\left(K+\mu C d_{1}\right)$. Also $u \in C^{4}[0,1] \cap C^{6}(0,1)$ is a solution of (13) if $u \in X$ satisfies $u=T\left(G u+h_{1}\right)$, where $h_{1}(t)=\mu u(t) \int_{0}^{1} G(t, s) u(s) d s+h(t)$, i.e.,

$$
u \in X, \quad(I-T G) u=T h_{1} .
$$

The operator $I-T G$ maps $X$ into $X$. From $\|T\| \leq D_{2}$ together with $\|G\| \leq\left(K+\mu C d_{1}\right)$ and the condition $D_{2}\left(K+\mu C d_{1}\right)<1$, and applying the operator spectral theorem, we find that $(I-T G)^{-1}$ exists and is bounded. Let $\mu \in\left(0, \frac{1-D_{2} K}{D_{2} C d_{1}}\right)$, where $1-D_{2} K>0$, then the condition $D_{2}\left(K+\mu C d_{1}\right)<1$ is fulfilled. Let $L=D_{2}\left(K+\mu C d_{1}\right)$, and let $\mu^{* *}=\frac{1-D_{2} K}{D_{2} C d_{1}}$.

Let $H=(I-T G)^{-1} T$. Then (16) is equivalent to $u=H h_{1}$. By the Neumann expansion formula, $H$ can be expressed by

$$
H=\left(I+T G+\cdots+(T G)^{n}+\cdots\right) T=T+(T G) T+\cdots+(T G)^{n} T+\cdots
$$

The complete continuity of $T$ with the continuity of $(I-T G)^{-1}$ guarantees that the operator $H: Y \rightarrow X$ is completely continuous. 
Now $\forall h \in Y_{+}$, let $u=T h$, then $u \in X \cap Y_{+}$, and $u^{\prime \prime} \leq 0, u^{(4)} \geq 0$. Thus, we have

$$
\begin{aligned}
(G u)(t)= & -(A(t)-a) u^{(4)}-(B(t)-b) u^{\prime \prime}-(C(t)-c) u \\
& +\mu D(t) \int_{0}^{1} G(t, s) u(s) d s \geq 0, \quad t \in[0,1] .
\end{aligned}
$$

Hence

$$
\forall h \in Y_{+}, \quad(G T h)(t) \geq 0, \quad t \in[0,1]
$$

and so, $(T G)(T h)(t)=T(G T h)(t) \geq 0, t \in[0,1]$.

It is easy to see [11] that the following inequalities hold: $\forall h \in Y_{+}$,

$$
\frac{1}{1-L}(T h)(t) \geq(H h)(t) \geq(T h)(t), \quad t \in[0,1],
$$

and, moreover,

$$
\|(H h)\|_{0} \leq \frac{1}{1-L}\|(T h)\|_{0}
$$

Lemma 6 [8] $H: Y \longrightarrow\left(X,\|\cdot\|_{\varkappa, v}\right)$ is completely continuous, where $\chi=\lambda_{1}+\lambda_{3}, v=$ $\lambda_{1} \lambda_{3}$ and $\forall h \in Y_{+}, \frac{1}{1-L}(T h)(t) \geq(H h)(t) \geq(T h)(t), t \in[0,1]$, and, moreover, $\|T h\|_{0} \geq$ $(1-L)\|H h\|_{0}$.

For any $u \in Y_{+}$, define $F u=\mu u(t) \int_{0}^{1} G(t, s) u(s) d s+\lambda f(t, u)$. From (H1), we have that $F$ : $Y_{+} \rightarrow Y_{+}$is continuous. It is easy to see that $u \in C^{4}[0,1] \cap C^{6}(0,1)$, being a positive solution of (13), is equivalent to $u \in Y_{+}$, being a nonzero solution of

$$
u=H F u \text {. }
$$

Let us introduce the following notations

$$
\begin{gathered}
T_{\lambda, \mu} u(t):=T F u(t)=\int_{0}^{1} \int_{0}^{1} \int_{0}^{1} G_{1}(t, v) G_{2}(v, s) G_{3}(s, \tau) \\
\times\left(\mu u(\tau) \int_{0}^{1} G(\tau, s) u(s) d s+\lambda f(\tau, u(\tau))\right) d \tau d s d \nu \\
Q_{\lambda, \mu} u:=H F u=T F u+(T G) T F u+(T G)^{2} T F u+\cdots+(T G)^{n} T F u+\cdots \\
=T_{\lambda, \mu} u+(T G) T_{\lambda, \mu} u+(T G)^{2} T_{\lambda, \mu} u+\cdots+(T G)^{n} T_{\lambda, \mu} u+\cdots
\end{gathered}
$$

i.e., $Q_{\lambda, \mu} u=H F u$. Obviously, $Q_{\lambda, \mu}: Y_{+} \rightarrow Y_{+}$is completely continuous. We next show that the operator $Q_{\lambda, \mu}$ has a nonzero fixed point in $Y_{+}$.

Let $P=\left\{u \in Y_{+}: u(t) \geq \delta_{1}(1-L) g_{1}(t)\|u(t)\|_{0}, t \in\left[\frac{1}{4}, \frac{3}{4}\right]\right\}$, where $g_{1}(t)=\frac{1}{C_{1}} G_{1}(t, t)$. It is easy to see that $P$ is a cone in $Y$, and now, we show $Q_{\lambda, \mu}(P) \subset P$.

Lemma $7 Q_{\lambda, \mu}(P) \subset P$ and $Q_{\lambda, \mu}: P \rightarrow P$ is completely continuous. 
Proof It is clear that $Q_{\lambda, \mu}: P \rightarrow P$ is completely continuous. Now $\forall u \in P$, let $h_{1}=F u$, then $h_{1} \in Y_{+}$. Using Lemma 6, i.e., $\left(Q_{\lambda, \mu} u\right)(t)=(H F u)(t) \geq(T F u)(t), t \in[0,1]$ and by Lemma 2, for all $u \in P$, we have

$$
(T F u)(t) \leq C_{1} \int_{0}^{1} \int_{0}^{1} \int_{0}^{1} G_{1}(\nu, v) G_{2}(\nu, s) G_{3}(s, \tau)(F u)(\tau) d \tau d s d \nu, \quad \forall t \in[0,1]
$$

Thus,

$$
\int_{0}^{1} \int_{0}^{1} \int_{0}^{1} G_{1}(v, v) G_{2}(v, s) G_{3}(s, \tau)(F u)(\tau) d \tau d s d v \geq \frac{1}{C_{1}}\|T F u\|_{0} .
$$

On the other hand, by Lemma 6 and (22), we have

$$
\begin{aligned}
(T F u)(t) & \geq \delta_{1} G_{1}(t, t) \int_{0}^{1} \int_{0}^{1} \int_{0}^{1} G_{1}(v, v) G_{2}(v, s) G_{3}(s, \tau)(F u)(\tau) d \tau d s d v \\
& \geq \delta_{1} G_{1}(t, t) \frac{1}{C_{1}}\|T F u\|_{0} \geq \delta_{1} G_{1}(t, t) \frac{1}{C_{1}}(1-L)\|Q u\|_{0}, \quad \forall t \in[0,1] .
\end{aligned}
$$

Thus, $Q_{\lambda, \mu}(P) \subset P$.

\section{Main results}

Lemma 8 Let $f(t, u)$ be nondecreasing in $u$ for $t \in[0,1]$ and $f(t, 0)>\widehat{c}>0$ for all $t \in[0,1]$, where $\widehat{c}$ is a constant and $L<1$. Then there exists $\lambda^{*}>0$ and $\mu^{*}>0$ such that the operator $Q_{\lambda, \mu}$ has a fixed point $u^{\star}$ at $\left(\lambda^{*}, \mu^{*}\right)$ with $u^{\star} \in P \backslash\{\theta\}$.

Proof Set $\widehat{u}_{1}(t)=\left(Q_{\lambda} *, \mu * u_{\star}\right)(t)$, where

$$
u_{\star}(t)=\frac{2}{1-L} \int_{0}^{1} \int_{0}^{1} \int_{0}^{1} G_{1}(t, v) G_{2}(v, \tau) G_{3}(\tau, s) d s d \tau d \nu
$$

It is easy to see that $u_{\star}(t) \in P$. Let $\lambda^{*}=M_{f u}^{-1}$ and $\mu^{*}=\min \left(N_{f u}^{-1} ; \mu^{* *}\right)$, where $M_{f u}=$ $\max _{t \in[0,1]} f\left(t, u_{\star}(t)\right)$ and $N_{f u}=\max _{t \in[0,1]} u_{\star}(t) \int_{0}^{1} G(t, s) u_{\star}(s) d s$, respectively. Then $M_{f u}>0$ and $N_{f u}>0$, and from Lemma 6 , we obtain

$$
\begin{aligned}
\widehat{u}_{0}(t)= & u_{\star}(t)=\frac{2}{1-L} \int_{0}^{1} \int_{0}^{1} \int_{0}^{1} G_{1}(t, v) G_{2}(v, \tau) G_{3}(\tau, s) d s d \tau d v \\
\geq & \frac{1}{1-L} \int_{0}^{1} \int_{0}^{1} \int_{0}^{1} G_{1}(t, v) G_{2}(v, \tau) G_{3}(\tau, s) \\
& \times\left(\lambda^{*} f\left(s, u_{\star}(s)\right)+\mu^{*} u_{\star}(t) \int_{0}^{1} G(t, s) u_{\star}(s) d s\right) d s d \tau d v \\
= & \frac{1}{1-L}\left(T_{\lambda *, \mu} u_{\star}\right)(t) \geq\left(Q_{\lambda} *, \mu * u_{\star}\right)(t)=\widehat{u}_{1}(t) .
\end{aligned}
$$

It is easy to see that

$$
\begin{aligned}
\widehat{u}_{n}(t) & =\left(Q_{\lambda *, \mu} * \widehat{u}_{n-1}\right)(t) \\
& =\left(T_{\lambda *, \mu} * \widehat{u}_{n-1}+(T G) T_{\lambda *, \mu} * \widehat{u}_{n-1}+(T G)^{2} T_{\lambda *, \mu} * \widehat{u}_{n-1}+\cdots\right.
\end{aligned}
$$




$$
\begin{aligned}
& \left.+(T G)^{n} T_{\lambda *, \mu} * \widehat{u}_{n-1}+\cdots\right) \\
\geq & \left(T_{\lambda *, \mu} * \widehat{u}_{n-2}+(T G) T_{\lambda *, \mu} * \widehat{u}_{n-2}+(T G)^{2} T_{\lambda *, \mu} * \widehat{u}_{n-2}+\cdots\right. \\
& \left.+(T G)^{n} T_{\lambda *, \mu} * \widehat{u}_{n-2}+\cdots\right)=\widehat{u}_{n-1}(t) .
\end{aligned}
$$

Indeed, for $h_{1}, h_{2} \in Y_{+}$, let $h_{1}(t) \geq h_{2}(t)$, then from (10), we have $u_{1}(t)=T h_{1} \geq T h_{2}=u_{2}(t)$. Using equation (4) and (6), we obtain

$$
-u^{\prime \prime}+\lambda_{2} u=\int_{0}^{1} \int_{0}^{1} G_{1}(t, v) G_{3}(v, \tau) h(\tau) d \tau d v, \quad t \in[0,1]
$$

and

$$
u^{(4)}-\left(\lambda_{2}+\lambda_{3}\right) u^{\prime \prime}+\lambda_{2} \lambda_{3} u=\int_{0}^{1} G_{1}(t, v) h(v) d v, \quad t \in[0,1] .
$$

Then by (23), we have for $t \in[0,1]$

$$
u_{1}^{\prime \prime}(t)-u_{2}^{\prime \prime}(t)=\lambda_{2}\left(u_{1}(t)-u_{2}(t)\right)-\int_{0}^{1} \int_{0}^{1} G_{1}(t, v) G_{3}(v, \tau)\left(h_{1}(t)-h_{2}(t)\right) d \tau d v \leq 0,
$$

because $\lambda_{2}<0$, and finally, from (24), we have

$$
\begin{aligned}
u_{1}^{(4)}(t)-u_{2}^{(4)}(t)= & \left(\lambda_{2}+\lambda_{3}\right)\left(u_{1}^{\prime \prime}(t)-u_{2}^{\prime \prime}(t)\right)-\lambda_{2} \lambda_{3}\left(u_{1}(t)-u_{2}(t)\right) \\
& +\int_{0}^{1} \int_{0}^{1} G_{1}(t, v)\left(h_{1}(t)-h_{2}(t)\right) d \tau d v \geq 0, \quad t \in[0,1]
\end{aligned}
$$

because $\lambda_{2}+\lambda_{3} \leq 0$ and $\lambda_{2} \lambda_{3} \leq 0$. From the equation

$$
\begin{aligned}
& (G u)(t)=-(A(t)-a) u^{(4)}-(B(t)-b) u^{\prime \prime}-(C(t)-c) u+\mu D(t) \int_{0}^{1} G(t, s) u(s) d s \geq 0, \\
& \quad t \in[0,1]
\end{aligned}
$$

we have

$$
\begin{aligned}
\left(G u_{1}\right)(t)-\left(G u_{2}\right)(t) & \\
= & -(A(t)-a)\left(u_{1}^{(4)}(t)-u_{2}^{(4)}(t)\right)-(B(t)-b)\left(u_{1}^{\prime \prime}(t)-u_{2}^{\prime \prime}(t)\right) \\
& -(C(t)-c)\left(u_{1}(t)-u_{2}(t)\right)+\mu D(t) \int_{0}^{1} G(t, s)\left(u_{1}(t)-u_{2}(t)\right) d s \geq 0, \\
& t \in[0,1]
\end{aligned}
$$

i.e., $\left(G u_{1}\right)(t) \geq\left(G u_{2}\right)(t)$ for all $t \in[0,1]$. Finally, if $h_{1}=F u_{1}$ and $h_{2}=F u_{2}$, then

$$
\begin{aligned}
\left(H h_{1}\right)(t) & =T\left(h_{1}\right)+(T G)\left(T h_{1}\right)+(T G)^{2}\left(T h_{1}\right)+\cdots+(T G)^{n}\left(T h_{1}\right)+\cdots \\
& \geq\left(H h_{2}\right)(t)=T\left(h_{2}\right)+(T G)\left(T h_{2}\right)+(T G)^{2}\left(T h_{2}\right)+\cdots+(T G)^{n}\left(T h_{2}\right)+\cdots
\end{aligned}
$$


i.e.,

$$
\begin{aligned}
Q_{\lambda} *, \mu & * u_{1} \\
& =\left(H F u_{1}\right)(t)=T\left(F u_{1}\right)+(T G)\left(T F u_{1}\right)+(T G)^{2}\left(T F u_{1}\right)+\cdots+(T G)^{n}\left(T F u_{1}\right)+\cdots \\
& \geq\left(H F u_{2}\right)(t)=T\left(F u_{2}\right)+(T G)\left(T F u_{2}\right)+(T G)^{2}\left(T F u_{2}\right)+\cdots+(T G)^{n}\left(T F u_{2}\right)+\cdots \\
& =Q_{\lambda *, \mu} * u_{2},
\end{aligned}
$$

and from (26), it follows that for $u_{1}, u_{2} \in Y_{+}$, if $u_{1}(t) \geq u_{2}(t)$ then, we have

$$
Q_{\lambda *, \mu} * u_{1} \geq Q_{\lambda *, \mu} * u_{2} .
$$

Set $\widehat{u}_{0}(t)=u_{\star}(t)$ and $\widehat{u}_{n}(t)=\left(Q_{\lambda^{\star}, \mu^{\star}} \widehat{u}_{n-1}\right)(t), n=1,2, \ldots, t \in[0,1]$. Then

$$
\widehat{u}_{0}(t)=u_{\star}(t) \geq \widehat{u}_{1}(t) \geq \cdots \geq \widehat{u}_{n}(t) \geq \cdots \geq L_{1} G_{1}(t, t)
$$

where

$$
L_{1}=\lambda^{*} \delta_{1} \delta_{2} \delta_{3} \widehat{c} C_{23} C_{12} C_{3} .
$$

Indeed, by Lemma 6, we have

$$
\begin{aligned}
\widehat{u}_{n}(t) & =Q_{\lambda *, \mu} * \widehat{u}_{n-1}=(H F)\left(\widehat{u}_{n-1}\right) \geq(T F)\left(\widehat{u}_{n-1}\right) \\
& \geq \lambda^{*} \int_{0}^{1} \int_{0}^{1} \int_{0}^{1} G_{1}(t, v) G_{2}(v, \tau) G_{3}(\tau, s) f\left(s, \widehat{u}_{n-1}(s)\right) d s d \tau d v \\
& \geq \lambda^{*} \widehat{c} \int_{0}^{1} \int_{0}^{1} \int_{0}^{1} G_{1}(t, v) G_{2}(v, \tau) G_{3}(\tau, s) d s d \tau d v \\
& \geq \lambda^{*} \widehat{c} \delta_{1} \delta_{2} \delta_{3} C_{12} C_{23} C_{3} G_{1}(t, t) .
\end{aligned}
$$

Now, $f(t, u)$ nondecreasing in $u$ for $t \in[0,1]$, Lemma 2, and the Lebesgue convergence theorem guarantee that $\left\{u_{n}\right\}_{n=0}^{\infty}=\left\{Q_{\lambda} *, * \widehat{u}_{0}\right\}_{n=0}^{\infty}$ decreases to a fixed point $u^{\star} \in P \backslash\{\theta\}$ of the operator $Q_{\lambda *, \mu} *$.

Lemma 9 Suppose that $(\mathrm{H} 3)-(\mathrm{H} 5)$ hold, and $L<1$. Set

$$
S_{\lambda, \mu}=\left\{u \in P: Q_{\lambda, \mu} u=u,(\lambda, \mu) \in A\right\},
$$

where $A \subset[a, \infty) \times[b, \infty)$ for some constants $a>0, b>0$. Then there exists a constant $C_{A}$ such that $\|u\|_{0}<C_{A}$ for all $u \in S_{\lambda, \mu}$.

Proof Suppose, to the contrary, that there exists a sequence $\left\{u_{n}\right\}_{n=1}^{\infty}$ such that $\lim _{n \rightarrow \infty}\left\|u_{n}\right\|_{0}=+\infty$, where $u_{n} \in P$ is a fixed point of the operator $Q_{\lambda, \mu}$ at $\left(\lambda_{n}, \mu_{n}\right) \in A$ $(n=1,2, \ldots)$. Then

$$
u_{n}(t) \geq k\left\|u_{n}\right\|_{0} \quad \text { for } t \in\left[\frac{1}{4}, \frac{3}{4}\right]
$$

where $k=\frac{\delta_{1}}{C_{1}}(1-L) \min _{t \in\left[\frac{1}{4}, \frac{3}{4}\right]} G_{1}(t, t)$. 
Choose $J_{1}>0$, so that

$$
J_{1} a \delta_{1} \delta_{2} \delta_{3} C_{12} C_{23} m_{1} m_{2} k>2,
$$

and $l_{1}>0$ such that

$$
f(t, u) \geq J_{1} u \quad \text { for } u>l_{1} \text { and } t \in\left[\frac{1}{4}, \frac{3}{4}\right]
$$

and $N_{0}$, so that $\left\|u_{N_{0}}\right\|>\frac{l_{1}}{k}$. Now,

$$
\begin{aligned}
\left(Q_{\lambda_{N_{0}}, \mu_{N_{0}}} u_{N_{0}}\right)\left(\frac{1}{2}\right) & \geq\left(T F u_{N_{0}}\right)\left(\frac{1}{2}\right) \\
& \geq \lambda_{N_{0}} \int_{0}^{1} \int_{0}^{1} \int_{0}^{1} G_{1}\left(\frac{1}{2}, v\right) G_{2}(v, \tau) G_{3}(\tau, s) f\left(s, u_{N_{0}}(s)\right) d s d \tau d v \\
& \geq \lambda_{N_{0}} \delta_{1} \delta_{2} \delta_{3} C_{12} C_{23} G_{1}\left(\frac{1}{2}, \frac{1}{2}\right) \int_{0}^{1} G_{3}(s, s) f\left(s, u_{N_{0}}(s)\right) d s \\
& \geq \lambda_{N_{0}} \delta_{1} \delta_{2} \delta_{3} C_{12} C_{23} G_{1}\left(\frac{1}{2}, \frac{1}{2}\right) \int_{\frac{1}{4}}^{\frac{3}{4}} G_{3}(s, s) f\left(s, u_{N_{0}}(s)\right) d s \\
& \geq \frac{1}{2} a \delta_{1} \delta_{2} \delta_{3} C_{12} C_{23} m_{1} m_{2} J_{1} u_{N_{0}}(t) \\
& \geq \frac{1}{2} a \delta_{1} \delta_{2} \delta_{3} C_{12} C_{23} m_{1} m_{2} J_{1} k\left\|u_{N_{0}}\right\|_{0}>\left\|u_{N_{0}}\right\|_{0}
\end{aligned}
$$

and so,

$$
\left\|u_{N_{0}}\right\|_{0}=\left\|Q_{\lambda_{N_{0}}, \mu_{N_{0}}} u_{N_{0}}\right\|_{0} \geq\left\|(T F) u_{N_{0}}\right\|_{0} \geq\left(T F u_{N_{0}}\right)\left(\frac{1}{2}\right)>\left\|u_{N_{0}}\right\|_{0}
$$

which is a contradiction.

Lemma 10 Suppose that $L<1,(\mathrm{H} 3)$ and $(\mathrm{H} 4)$ hold and that the operator $Q_{\lambda, \mu}$ has a positive fixed point in $P$ at $\widehat{\lambda}>0$ and $\widehat{\mu}>0$. Then for every $\left(\lambda_{\star}, \mu_{\star}\right) \in(0, \widehat{\lambda}) \times(0, \widehat{\mu})$ there exists a function $u_{\star} \in P \backslash\{\theta\}$ such that $Q_{\lambda_{\star}, \mu_{\star}} u_{\star}=u_{\star}$.

Proof Let $\widehat{u}(t)$ be a fixed point of the operator $Q_{\lambda, \mu}$ at $\left.\widehat{\lambda}, \widehat{\mu}\right)$. Then

$$
\widehat{u}(t)=Q_{\widehat{\lambda}, \widehat{\mu}} \widehat{u}(t) \geq Q_{\lambda_{\star}, \mu_{\star}} \widehat{u}(t),
$$

where $0<\lambda_{\star}<\widehat{\lambda}, 0<\mu_{\star}<\widehat{\mu}$. Hence

$$
\begin{aligned}
\int_{0}^{1} & \int_{0}^{1} \int_{0}^{1} G_{1}(t, v) G_{2}(v, \tau) G_{3}(\tau, s)\left(\widehat{\lambda} f(s, \widehat{u}(s))+\widehat{\mu} \widehat{u}(s) \int_{0}^{1} G(s, p) \widehat{u}(p) d p\right) d s d \tau d v \\
\geq & \int_{0}^{1} \int_{0}^{1} \int_{0}^{1} G_{1}(t, v) G_{2}(v, \tau) G_{3}(\tau, s) \\
& \times\left(\lambda_{\star} f(s, \widehat{u}(s))+\mu_{\star} \widehat{u}(s) \int_{0}^{1} G(s, p) \widehat{u}(p) d p\right) d s d \tau d v .
\end{aligned}
$$


Set

$$
\begin{aligned}
\left(T_{\lambda_{\star}, \mu_{\star}} u\right)(t)= & \int_{0}^{1} \int_{0}^{1} \int_{0}^{1} G_{1}(t, v) G_{2}(v, \tau) G_{3}(\tau, s) \\
& \times\left(\lambda_{\star} f(s, u(s))+\mu_{\star} u(s) \int_{0}^{1} G(s, p) u(p) d p\right) d s d \tau d v
\end{aligned}
$$

and

$$
\left(Q_{\lambda_{\star}, \mu_{\star}} u\right)(t)=T_{\lambda_{\star}, \mu_{\star}} u+(T G) T_{\lambda_{\star}, \mu_{\star}} u+(T G)^{2} T_{\lambda_{\star}, \mu_{\star}} u+\cdots+(T G)^{n} T_{\lambda_{\star}, \mu_{\star}} u+\cdots
$$

$u_{0}(t)=\widehat{u}(t)$ and $u_{n}(t)=Q_{\lambda_{\star}, \mu_{\star}} u_{n-1}$. Then

$$
\begin{aligned}
u_{0}(t) & =\widehat{u}(t)=T_{\widehat{\lambda}, \widehat{u}} \widehat{u}+(T G) T_{\widehat{\lambda}, \widehat{u}} \widehat{u}+(T G)^{2} T_{\widehat{\lambda}, \widehat{\mu}} \widehat{u}+\cdots+(T G)^{n} T_{\widehat{\lambda}, \widehat{\mu}} \widehat{u}+\cdots \\
& \geq T_{\lambda_{\star}, \mu_{\star}} \widehat{u}+(T G) T_{\lambda_{\star}, \mu_{\star}} \widehat{u}+(T G)^{2} T_{\lambda_{\star}, \mu_{\star}} \widehat{u}+\cdots+(T G)^{n} T_{\lambda_{\star}, \mu_{\star}} \widehat{u}+\cdots=u_{1}(t)
\end{aligned}
$$

and

$$
\begin{aligned}
u_{n}(t)=Q_{\lambda_{\star}, \mu_{\star}} u_{n-1}= & T_{\lambda_{\star}, \mu_{\star}} u_{n-1}+(T G) T_{\lambda_{\star}, \mu_{\star}} u_{n-1}+(T G)^{2} T_{\lambda_{\star}, \mu_{\star}} u_{n-1}+\cdots \\
& +(T G)^{n} T_{\lambda_{\star}, \mu_{\star}} u_{n-1}+\cdots \\
\geq & T_{\lambda_{\star}, \mu_{\star}} u_{n-2}+(T G) T_{\lambda_{\star}, \mu_{\star}} u_{n-2}+(T G)^{2} T_{\lambda_{\star}, \mu_{\star}} u_{n-2}+\cdots \\
& +(T G)^{n} T_{\lambda_{\star}, \mu_{\star}} u_{n-2}+\cdots=u_{n-1}(t)
\end{aligned}
$$

because $f(t, u)$ is nondecreasing in $u$ for $t \in[0,1]$ and $T_{\lambda_{\star}, \mu_{\star}} u$ is also nondecreasing in $u$. Thus

$$
u_{0}(t) \geq u_{1}(t) \geq \cdots \geq u_{n}(t) \geq u_{n+1}(t) \geq \cdots \geq L_{2} G_{1}(t, t)
$$

where

$$
L_{2}=\lambda * \widehat{c} \delta_{1} \delta_{2} \delta_{3} C_{12} C_{23} C_{3} \text {. }
$$

Indeed, by Lemma 6, we have

$$
\begin{aligned}
u_{n}(t) & =Q_{\lambda_{\star}, \mu_{\star}} u_{n-1}=(H F)\left(u_{n-1}\right) \geq T_{\lambda_{\star}, \mu_{\star}}\left(u_{n-1}\right) \\
& \geq \lambda_{\star} \int_{0}^{1} \int_{0}^{1} \int_{0}^{1} G_{1}(t, v) G_{2}(v, \tau) G_{3}(\tau, s) f\left(s, u_{n-1}(s)\right) d s d \tau d v \\
& \geq \lambda_{\star} \widehat{c} \int_{0}^{1} \int_{0}^{1} \int_{0}^{1} G_{1}(t, v) G_{2}(v, \tau) G_{3}(\tau, s) d s d \tau d v \geq \lambda_{\star} \widehat{c} \delta_{1} \delta_{2} \delta_{3} C_{12} C_{23} C_{3} G_{1}(t, t) .
\end{aligned}
$$

Lemma 2 implies that $\left\{Q_{\lambda^{\star}}^{n} u\right\}_{n=1}^{\infty}$ decreases to a fixed point $u_{\star} \in P \backslash\{\theta\}$.

Lemma 11 Suppose that $L<1$, (H3)-(H5) hold. Let

$$
\Lambda=\left\{\lambda>0, \mu>0: Q_{\lambda, \mu} \text { have at least one fixed point at }(\lambda, \mu) \text { in } P\right\} .
$$


Proof Suppose, to the contrary, that there exists a fixed point sequence $\left\{u_{n}\right\}_{n=0}^{\infty} \subset P$ of $Q_{\lambda, \mu}$ at $\left(\lambda_{n}, \mu_{n}\right)$ such that $\lim _{n \rightarrow \infty} \lambda_{n}=\infty$ and $0<\mu_{n}<\mu^{* *}$. Then there are two cases to be considered: (i) there exists a subsequence $\left\{u_{n_{i}}\right\}_{n=0}^{\infty}$ such that $\lim _{i \rightarrow \infty}\left\|u_{n_{i}}\right\|_{0}=\infty$, which is impossible by Lemma 9, so we only consider the next case: (ii) there exists a constant $H>0$ such that $\left\|u_{n}\right\|_{0} \leq H, n=0,1,2,3, \ldots$. In view of (H3) and (H4), we can choose $l_{0}>0$ such that $f(t, 0)>l_{0} H$, and further, $f\left(t, u_{n}\right)>l_{0} H$ for $t \in[0,1]$. We know that

$$
u_{n}=Q_{\lambda_{n}, \mu_{n}} u_{n} \geq T_{\lambda_{n}, \mu_{n}} u_{n}
$$

Let $v_{n}(t)=T_{\lambda_{n}, \mu_{n}} u_{n}$, i.e., $u_{n}(t) \geq v_{n}(t)$. Then it follows that

$$
-v_{n}^{(6)}+a v_{n}^{(4)}+b v_{n}^{\prime \prime}+c v_{n}=\lambda_{n} f\left(t, u_{n}\right)+\mu_{n} u_{n}(t) \int_{0}^{1} G(t, p) u_{n}(p) d p, \quad 0<t<1 .
$$

Multiplying (29) by $\sin \pi t$ and integrating over [0,1], and then using integration by parts on the left side of (29), we have

$$
\Gamma \int_{0}^{1} v_{n}(t) \sin \pi t d t=\lambda_{n} \int_{0}^{1} f\left(t, u_{n}\right) \sin \pi t d t+\mu_{n} \int_{0}^{1} u_{n}(t) \sin \pi t \int_{0}^{1} G(t, p) u_{n}(p) d p d t .
$$

Next, assume that (ii) holds. Then

$$
\begin{aligned}
& \Gamma \int_{0}^{1} u_{n}(t) \sin \pi t d t \geq \Gamma \int_{0}^{1} v_{n}(t) \sin \pi t d t \\
& =\lambda_{n} \int_{0}^{1} f\left(t, u_{n}\right) \sin \pi t d t+\mu_{n} \int_{0}^{1} u_{n}(t) \sin \pi t \int_{0}^{1} G(t, p) u_{n}(p) d p d t
\end{aligned}
$$

and

$$
\begin{aligned}
\Gamma H \int_{0}^{1} \sin \pi t d t & \geq \Gamma\left\|u_{n}\right\|_{0} \int_{0}^{1} \sin \pi t d t \geq \Gamma \int_{0}^{1} u_{n}(t) \sin \pi t d t \geq \Gamma \int_{0}^{1} v_{n}(t) \sin \pi t d t \\
& =\lambda_{n} \int_{0}^{1} f\left(t, u_{n}\right) \sin \pi t d t+\mu_{n} \int_{0}^{1} u_{n}(t) \sin \pi t \int_{0}^{1} G(t, p) u_{n}(p) d p d t \\
& \geq \lambda_{n} l_{0} H \int_{0}^{1} \sin \pi t d t
\end{aligned}
$$

lead to $\Gamma \geq \lambda_{n} l_{0}$, which is a contradiction. The proof is complete.

Lemma 12 Suppose that $L<1$, (H3)-(H4) hold. Let

$$
\Lambda_{\mu}=\{\lambda>0:(\lambda, \mu) \in \Lambda \text { and } \mu \text { is fixed }\}
$$

and let $\tilde{\lambda}_{\mu}=\sup \Lambda_{\mu}$. Then $\Lambda_{\mu}=\left(0, \widetilde{\lambda}_{\mu}\right]$, where $\Lambda$ is defined in Lemma 11 .

Proof By Lemma 10, it follows that $(0, \tilde{\lambda}) \times(0, \mu) \subset \Lambda$. We only need to prove $\left(\tilde{\lambda}_{\mu}, \mu\right) \in \Lambda$. We may choose a distinct nondecreasing sequence $\left\{\lambda_{n}\right\}_{n=1}^{\infty} \subset \Lambda$ such that $\lim _{n \rightarrow \infty} \lambda_{n}=\tilde{\lambda}_{\mu}$. Set $u_{n} \in P$ as a fixed point of $Q_{\lambda, \mu}$ at $\left(\lambda_{n}, \mu\right), n=1,2, \ldots$, i.e., $u_{n}=Q_{\lambda_{n}, \mu} u_{n}$. By Lemma 9, 
$\left\{u_{n}\right\}_{n=1}^{\infty}$, is uniformly bounded, so it has a subsequence, denoted by $\left\{u_{n_{k}}\right\}_{k=1}^{\infty}$, converging to $\tilde{u} \in P$. Note that

$$
\begin{aligned}
u_{n} & =T_{\lambda_{n}, \mu} u_{n}+(T G) T_{\lambda_{n}, \mu} u_{n}+(T G)^{2} T_{\lambda_{n}, \mu} u_{n}+\cdots+(T G)^{n} T_{\lambda_{n}, \mu} u_{n}+\cdots \\
& =Q_{\lambda_{n}, \mu} u_{n} .
\end{aligned}
$$

Taking the limit as $n \rightarrow \infty$ on both sides of (30), and using the Lebesgue convergence theorem, we have

$$
\widetilde{u}=T_{\tilde{\lambda}, \mu} \widetilde{u}+(T G) T_{\tilde{\lambda}, \mu} \widetilde{u}_{n}+(T G)^{2} T_{\tilde{\lambda}, \mu} \widetilde{u}+\cdots+(T G)^{n} T_{\tilde{\lambda}, \mu} \widetilde{u}+\cdots
$$

which shows that $Q_{\lambda, \mu}$ has a positive fixed point $\tilde{u}$ at $(\tilde{\lambda}, \mu)$.

Theorem 1 Suppose that (H3)-(H5) hold, and $L<1$. For fixed $\mu^{\star} \in\left(0, \mu^{\star \star}\right)$, then there exists at $\lambda^{\star}>0$ such that (3) has at least two, one and has no positive solutions for $0<\lambda<\lambda^{\star}$, $\lambda=\lambda^{\star}$ for $\lambda>\lambda^{\star}$, respectively.

Proof Suppose that (H3) and (H4) hold. Then there exists $\lambda^{\star}>0$ and $\mu^{\star}>0$ such that $Q_{\lambda, \mu}$ has a fixed point $u_{\lambda^{\star}, \mu^{\star}} \in P \backslash\{\theta\}$ at $\lambda=\lambda^{\star}$ and $\mu=\mu^{\star}$. In view of Lemma 12, $Q_{\lambda, \mu}$ also has a fixed point $u_{\underline{\lambda}, \underline{\mu}}<u_{\lambda^{\star}, \mu^{\star}}, u_{\underline{\lambda}, \underline{\mu}} \in P \backslash\{\theta\}$, and $0<\underline{\lambda}<\lambda^{\star}, 0<\underline{\mu}<\mu^{\star}, \mu^{\star} \in\left(0, \mu^{\star \star}\right)$. For $0<\underline{\lambda}<\lambda^{\star} \backslash$, there exists $\delta_{0}>0$ such that

$$
f\left(t, u_{\lambda^{\star}, \mu^{\star}}+\delta\right)-f\left(t, u_{\lambda^{\star}, \mu^{\star}}\right) \leq f(t, 0)\left(\frac{\lambda^{\star}}{\underline{\lambda}}-1\right)
$$

for $t \in[0,1], 0<\delta \leq \delta_{0}$. In this case, it is easy to see that

$$
\begin{aligned}
T_{\underline{\lambda}, \underline{\mu}}\left(u_{\lambda^{\star}, \mu^{\star},}+\delta\right)= & \underline{\lambda} \int_{0}^{1} \int_{0}^{1} \int_{0}^{1} G_{1}(t, v) G_{2}(v, \tau) G_{3}(\tau, s) f\left(s, u_{\lambda^{\star}, \mu^{\star}}(s)+\delta\right) d s d \tau d v \\
& +\underline{\mu} \int_{0}^{1} \int_{0}^{1} \int_{0}^{1} G_{1}(t, v) G_{2}(v, \tau) G_{3}(\tau, s)\left(u_{\lambda^{\star}, \mu^{\star}}(s)+\delta\right) \\
& \times \int_{0}^{1} G(s, p)\left(u_{\lambda^{\star}, \mu^{\star}}(p)+\delta\right) d p d s d \tau d v \\
\leq & \lambda^{\star} \int_{0}^{1} \int_{0}^{1} \int_{0}^{1} G_{1}(t, v) G_{2}(v, \tau) G_{3}(\tau, s) f\left(s, u_{\lambda^{\star}}(s)\right) d s d \tau d v \\
& +\mu^{\star} \int_{0}^{1} \int_{0}^{1} \int_{0}^{1} G_{1}(t, v) G_{2}(v, \tau) G_{3}(\tau, s) u_{\lambda^{\star}, \mu^{\star}}(s) \\
& \times \int_{0}^{1} G(s, p) u_{\lambda^{\star}, \mu^{\star}}(p) d p d s d \tau d v=T_{\lambda^{\star}, \mu^{\star}} u_{\lambda^{\star}, \mu^{\star}} .
\end{aligned}
$$

Indeed, we have

$$
\begin{aligned}
\underline{\lambda} \int_{0}^{1} & \int_{0}^{1} \int_{0}^{1} G_{1}(t, v) G_{2}(v, \tau) G_{3}(\tau, s) f\left(s, u_{\lambda^{\star}}(s)+\delta\right) d s d \tau d v \\
& \quad-\lambda^{\star} \int_{0}^{1} \int_{0}^{1} \int_{0}^{1} G_{1}(t, v) G_{2}(v, \tau) G_{3}(\tau, s) f\left(s, u_{\lambda^{\star}}(s)\right) d s d \tau d v
\end{aligned}
$$




$$
\begin{aligned}
= & \underline{\lambda} \int_{0}^{1} \int_{0}^{1} \int_{0}^{1} G_{1}(t, v) G_{2}(v, \tau) G_{3}(\tau, s)\left\{f\left(s, u_{\lambda^{\star}}(s)+\delta\right)-f\left(s, u_{\lambda^{\star}}(s)\right)\right\} d s d \tau d v \\
& -\left(\lambda^{\star}-\underline{\lambda}\right) \int_{0}^{1} \int_{0}^{1} \int_{0}^{1} G_{1}(t, v) G_{2}(v, \tau) G_{3}(\tau, s) f\left(s, u_{\lambda^{\star}}(s)\right) d s d \tau d v \\
\leq & \left(\lambda^{\star}-\underline{\lambda}\right) \int_{0}^{1} \int_{0}^{1} \int_{0}^{1} G_{1}(t, v) G_{2}(v, \tau) G_{3}(\tau, s) f(s, 0) d s d \tau d v \\
& -\left(\lambda^{\star}-\underline{\lambda}\right) \int_{0}^{1} \int_{0}^{1} \int_{0}^{1} G_{1}(t, v) G_{2}(v, \tau) G_{3}(\tau, s) f\left(s, u_{\lambda^{\star}}(s)\right) d s d \tau d v \\
= & \left(\lambda^{\star}-\underline{\lambda}\right) \int_{0}^{1} \int_{0}^{1} \int_{0}^{1} G_{1}(t, v) G_{2}(v, \tau) G_{3}(\tau, s)\left\{f(s, 0)-f\left(s, u_{\lambda^{\star}}(s)\right)\right\} d s d \tau d v \leq 0 .
\end{aligned}
$$

Similarly, it is easy to see that

$$
\begin{aligned}
& \underline{\mu} \int_{0}^{1} \int_{0}^{1} \int_{0}^{1} G_{1}(t, v) G_{2}(v, \tau) G_{3}(\tau, s)\left(u_{\lambda^{\star}, \mu^{\star}}(s)+\delta\right) \int_{0}^{1} G(s, p)\left(u_{\lambda^{\star}, \mu^{\star}}(p)+\delta\right) d p d s d \tau d \nu \\
& \quad-\mu^{\star} \int_{0}^{1} \int_{0}^{1} \int_{0}^{1} G_{1}(t, v) G_{2}(v, \tau) G_{3}(\tau, s) u_{\lambda^{\star}, \mu^{\star}}(s) \int_{0}^{1} G(s, p) u_{\lambda^{\star}, \mu^{\star}}(p) d p d s d \tau d \nu \leq 0 .
\end{aligned}
$$

Moreover, from (25), it follows that for $T_{\underline{\lambda}, \underline{\mu}}\left(u_{\lambda^{\star}, \mu^{\star}}+\delta\right) \leq T_{\lambda^{\star}, \mu^{\star}} u_{\lambda^{\star}, \mu^{\star}}$ we have

$$
G\left(T_{\underline{\lambda}, \underline{\mu}}\left(u_{\lambda^{\star}, \mu^{\star}}+\delta\right)\right) \leq G\left(T_{\lambda^{\star}, \mu^{\star}} u_{\lambda^{\star}, \mu^{\star}}\right) .
$$

Finally, we have

$$
(T G) T_{\underline{\lambda}, \underline{\mu}}\left(u_{\lambda^{\star}, \mu^{\star}}+\delta\right) \leq(T G) T_{\lambda^{\star}, \mu^{\star}} u_{\lambda^{\star}, \mu^{\star}} .
$$

By induction, it is easy to see that

$$
(T G)^{n} T_{\underline{\lambda}, \underline{\mu}}\left(u_{\lambda^{\star}, \mu^{\star}}+\delta\right) \leq(T G)^{n} T_{\lambda^{\star}, \mu^{\star}} u_{\lambda^{\star}, \mu^{\star}}, \quad n=1,2, \ldots
$$

Hence, using (31), we have

$$
\begin{aligned}
Q_{\underline{\lambda}, \underline{\mu}}\left(u_{\lambda^{\star}, \mu^{\star}}+\delta\right)= & T_{\underline{\lambda}, \underline{\mu}}\left(u_{\lambda^{\star}, \mu^{\star}}+\delta\right)+(T G) T_{\underline{\lambda}, \underline{\mu}}\left(u_{\lambda^{\star}, \mu^{\star}}+\delta\right) \\
& +(T G)^{2} T_{\underline{\lambda}, \underline{\mu}}\left(u_{\lambda^{\star}, \mu^{\star}}+\delta\right)+\cdots+(T G)^{n} T_{\underline{\lambda}, \mu}\left(u_{\lambda^{\star}, \mu^{\star}}+\delta\right)+\cdots \\
\leq & T_{\lambda^{\star}, \mu^{\star}} u_{\lambda^{\star}, \mu^{\star}}+(T G) T_{\lambda^{\star}, \mu^{\star}} u_{\lambda^{\star}, \mu^{\star}}+(T G)^{2} T_{\lambda^{\star}, \mu^{\star}} u_{\lambda^{\star}, \mu^{\star}}+\cdots \\
& +(T G)^{n} T_{\lambda^{\star}, \mu^{\star}} u_{\lambda^{\star}, \mu^{\star}}+\cdots \\
= & Q_{\lambda^{\star}, \mu^{\star}}\left(u_{\lambda^{\star}, \mu^{\star}}\right)
\end{aligned}
$$

i.e.,

$$
Q_{\underline{\lambda}, \underline{\mu}}\left(u_{\lambda^{\star}, \mu^{\star}}+\delta\right)-Q_{\lambda^{\star}, \mu^{\star}}\left(u_{\lambda^{\star}, \mu^{\star}}\right) \leq 0,
$$

so that

$$
Q_{\underline{\lambda}, \underline{\mu}}\left(u_{\lambda^{\star}, \mu^{\star}}+\delta\right) \leq Q_{\lambda^{\star}, \mu^{\star}}\left(u_{\lambda^{\star}, \mu^{\star}}\right)=u_{\lambda^{\star}, \mu^{\star}}<u_{\lambda^{\star}, \mu^{\star}}+\delta .
$$


Set $D_{u_{\lambda^{\star}, \mu^{\star}}}=\left\{u \in C[0,1]:-\delta<u(t)<u_{\lambda^{\star}, \mu^{\star}}+\delta\right\}$. Then $Q_{\underline{\lambda}, \underline{\mu}}: P \cap D_{u_{\lambda^{\star}, \mu^{\star}}} \rightarrow P$ is completely continuous. Furthermore, $Q_{\underline{\lambda}, \underline{\mu}} u \neq v u$ for $v \geq 1$ and $\bar{u} \in P \cap \partial D_{u_{\lambda^{\star}, \mu^{\star}}}$. Indeed set $u \in P \cap \partial D_{u_{\lambda^{\star}, \mu^{\star}}}$. Then there exists $t_{0} \in[0,1]$ such that $u\left(t_{0}\right)=\|u\|_{0}=\left\|u_{\lambda^{\star}, \mu^{\star}}+\delta\right\|_{0}$ and

$$
\begin{aligned}
\left(Q_{\underline{\lambda}, \underline{\mu}} u\right)\left(t_{0}\right)= & \left(T_{\underline{\lambda}, \underline{\mu}}(u)+(T G) T_{\underline{\lambda} \underline{\mu}}(u)+(T G)^{2} T_{\underline{\lambda}, \underline{\mu}}(u)+\cdots+(T G)^{n} T_{\underline{\lambda}, \underline{\mu}}(u)+\cdots\right)\left(t_{0}\right) \\
\leq & \left(T_{\underline{\lambda}, \underline{\mu}}\left(u_{\lambda^{\star}, \mu^{\star}}+\delta\right)+(T G) T_{\underline{\lambda}, \underline{\mu}}\left(u_{\lambda^{\star}, \mu^{\star}}+\delta\right)+(T G)^{2} T_{\underline{\lambda}, \underline{\mu}}\left(u_{\lambda^{\star}, \mu^{\star}}+\delta\right)+\cdots\right. \\
& \left.+(T G)^{n} T_{\underline{\lambda}, \underline{\mu}}\left(u_{\lambda^{\star}, \mu^{\star}}+\delta\right)+\cdots\right)\left(t_{0}\right)=Q_{\underline{\lambda}, \underline{\mu}}\left(u_{\lambda^{\star}, \mu^{\star}}+\delta\right)\left(t_{0}\right) \\
& <u_{\lambda^{\star}, \mu^{\star}}\left(t_{0}\right)+\delta=u\left(t_{0}\right) \leq v u\left(t_{0}\right), \quad v \geq 1 .
\end{aligned}
$$

By Lemma 3, $i\left(Q_{\underline{\lambda}, \underline{\mu}}, P \cap \partial D_{u_{\lambda^{\star}, \mu^{\star}}}, P\right)=1$.

Let $k$ be such that

$$
u(t) \geq k\|u\|_{0} \quad \text { for } t \in\left[\frac{1}{4}, \frac{3}{4}\right] .
$$

We know that $\lim _{u \rightarrow \infty} \frac{f(t, u)}{u}=\infty$ uniformly for $t \in[0,1]$, so we may choose $J_{3}>0$, so that

$$
\underline{\lambda}_{3} \delta_{1} \delta_{2} \delta_{3} C_{12} C_{23} m_{1} C_{3} k>2 \text {, }
$$

$l_{3}>\left\|u_{\lambda^{\star}, \mu^{\star}}+\delta\right\|_{0}>0$, so that

$$
f(t, u) \geq J_{3} u \quad \text { for } u>l_{3} \text { and } t \in\left[\frac{1}{4}, \frac{3}{4}\right]
$$

Set $R_{1}=\frac{l_{3}}{k}$ and $P_{R_{1}}=\left\{u \in P:\|u\|_{0}<R_{1}\right\}$. Then $Q_{\underline{\lambda}} \underline{\mu}: \bar{P}_{R_{1}} \rightarrow P$ is completely continuous. It is easy to obtain

$$
\begin{aligned}
\left(Q_{\underline{\lambda}, \underline{\mu}} u\right)(t) & \geq\left(T_{\underline{\lambda}, \underline{\mu}} u\right)(t) \geq \underline{\lambda} \int_{0}^{1} \int_{0}^{1} \int_{0}^{1} G_{1}(t, v) G_{2}(v, \tau) G_{3}(\tau, s) f(s, u(s)) d s d \tau d v \\
& \geq \underline{\lambda} \delta_{1} \delta_{2} \delta_{3} C_{12} C_{23} G_{1}(t, t) \int_{0}^{1} G_{3}(s, s) f(s, u(s)) d s \\
& \geq \underline{\lambda} \delta_{1} \delta_{2} \delta_{3} C_{12} C_{23} G_{1}(t, t) \int_{\frac{1}{4}}^{\frac{3}{4}} G_{3}(s, s) f(s, u(s)) d s \\
& \geq \frac{1}{2} \underline{\lambda} \delta_{1} \delta_{2} \delta_{3} C_{12} C_{23} m_{1} C_{3} J_{3} u(t) \geq \frac{1}{2} \underline{\lambda} \delta_{1} \delta_{2} \delta_{3} C_{12} C_{23} m_{1} C_{3} J_{3} k\|u\|_{0}>\|u\|_{0}
\end{aligned}
$$

for $t \in[0,1]$ and $u \in \partial P_{R_{1}}$. Now $u(t) \geq k\|u\|_{0}=k R_{1}=l_{3}$, and so

$$
\left\|Q_{\underline{\lambda}, \underline{\mu}} u\right\|_{0}>\|u\|_{0} .
$$

In view of Lemma $4, i\left(Q_{\underline{\lambda}, \underline{\mu}}, P_{R_{1}}, P\right)=0$. By the additivity of the fixed point index,

$$
i\left(Q_{\underline{\lambda}, \underline{\mu}}, P_{R_{1}} \backslash{\overline{P \cap D_{u_{\lambda^{\star}, \mu^{\star}}}}} P\right)=i\left(Q_{\underline{\lambda}, \underline{\mu}}, P_{R_{1}}, P\right)-i\left(Q_{\underline{\lambda}, \underline{\mu}}, P \cap D_{u_{\lambda^{\star}, \mu^{\star}}}, P\right)=-1 .
$$

Thus $Q_{\underline{\lambda}, \underline{\mu}}$ has a fixed point in $\left\{P \cap D_{u_{\lambda^{\star}, \mu^{\star}}}\right\} \backslash\{\theta\}$ and has another fixed point in $P_{R_{1}} \backslash P \cap$ $D_{u_{\lambda^{\star}, \mu^{\star}}}$ by choosing $\lambda^{\star}=\tilde{\lambda}$. 
Let us introduce the notation $\mu=0$ in the equation of (13), then we have

$$
\begin{aligned}
& -u^{(6)}+A(t) u^{(4)}+B(t) u^{\prime \prime}+C(t) u=\lambda f(t, u), \\
& u(0)=u(1)=u^{\prime \prime}(0)=u^{\prime \prime}(1)=u^{(4)}(0)=u^{(4)}(1)=0 .
\end{aligned}
$$

In this case, we can prove the following theorem, which is similar to Theorem 1.

Theorem 2 Suppose that (H3)-(H5) hold, and $L<1$. Then there exists at $\lambda^{\star}>0$ such that (32) has at least two, one and has no positive solutions for $0<\lambda<\lambda^{\star}, \lambda=\lambda^{\star}$ for $\lambda>\lambda^{\star}$, respectively.

We follow exactly the same procedure, described in detail in the proof of Theorem 1 for $\mu=0$.

Let us introduce the following notations for $\mu=0$ and $\lambda=1$

$$
\begin{gathered}
T F u(t)=\int_{0}^{1} \int_{0}^{1} \int_{0}^{1} G_{1}(t, v) G_{2}(v, s) G_{3}(s, \tau) f(\tau, u(\tau)) d \tau d s d v, \\
Q u:=H F u=T F u+(T G) T F u+(T G)^{2} T F u+\cdots+(T G)^{n} T F u+\cdots, \\
\text { i.e., } Q u=Q_{1,0} u=H F u .
\end{gathered}
$$

Lemma 13 Suppose that (H3), (H4) and (H6) hold, and $L<1$. Then for any $u \in$ $C^{+}[0,1] \backslash\{\theta\}$, there exist real numbers $S_{u} \geq s_{u}>0$ such that

$$
s_{u} g(t) \leq(Q u)(t) \leq S_{u} g(t), \quad \text { for } t \in[0,1]
$$

where $g(t)=\int_{0}^{1} \int_{0}^{1} G_{1}(t, \tau) G_{2}(\tau, v) G_{3}(v, v) d v d \tau$.

Proof For any $u \in C^{+}[0,1] \backslash\{\theta\}$ from Lemma 6, we have

$$
\begin{aligned}
(Q u)(t)=(H F u)(t) & \leq \frac{1}{1-L} \int_{0}^{1} \int_{0}^{1} \int_{0}^{1} G_{1}(t, v) G_{2}(v, \tau) G_{3}(\tau, s) f(s, u(s)) d s d \tau d v \\
& \leq \frac{C_{3}}{1-L} \max _{s \in[0,1]} f(s, u(s)) \int_{0}^{1} \int_{0}^{1} G_{1}(t, \tau) G_{2}(\tau, v) G_{3}(v, v) d v d \tau \\
& =\frac{C_{3}}{1-L} \max _{s \in[0,1]} f(s, u(s)) g(t)=S_{u} g(t) \quad \text { for } t \in[0,1] .
\end{aligned}
$$

Note that for any $u \in C^{+}[0,1] \backslash\{\theta\}$, there exists an interval $\left[a_{1}, b_{1}\right] \subset(0,1)$ and a number $p>0$ such that $u(t) \geq p$ for $t \in\left[a_{1}, b_{1}\right]$. In addition, by (H6), there exists $s_{0}>0$ and $u^{0} \in$ $(0, \infty)$ such that $f\left(t, u^{0}\right) \geq s_{0}$ for $t \in\left[a_{1}, b_{1}\right]$. If $p \geq u^{0}$, then $f(t, u) \geq f(t, p) \geq f\left(t, u^{0}\right) \geq s_{0}$; if $p<u^{0}$, then $f(t, u) \geq f(t, p) \geq f\left(t, \frac{p}{u^{0}} p\right) \geq\left(\frac{p}{u^{0}}\right)^{\alpha} s_{0}$. Hence

$$
\begin{aligned}
(Q u)(t) & \geq(T F u)(t) \\
& =\int_{0}^{1} \int_{0}^{1} \int_{0}^{1} G_{1}(t, v) G_{2}(v, \tau) G_{3}(\tau, s) f(s, u(s)) d s d \tau d v \\
& \geq \delta_{3} \int_{0}^{1} \int_{0}^{1} \int_{0}^{1} G_{1}(t, v) G_{2}(v, \tau) G_{3}(\tau, \tau) G_{3}(s, s) f(s, u(s)) d s d \tau d v
\end{aligned}
$$




$$
\begin{aligned}
& \geq \delta_{3} g(t) \int_{a_{1}}^{b_{1}} G_{3}(s, s) f(s, u(s)) d s d \tau d v \\
& \geq\left(b_{1}-a_{1}\right) \delta_{3} g(t) m_{G}\left(\frac{p}{u^{0}}\right)^{\alpha}=s_{u} g(t),
\end{aligned}
$$

where $m_{G}=\min _{s \in\left[a_{1}, b_{1}\right]} G_{3}(s, s), g(t)=\int_{0}^{1} \int_{0}^{1} G_{1}(t, v) G_{2}(v, \tau) G_{3}(\tau, \tau) d \tau d v, s_{u}=\left(b_{1}-a_{1}\right) \times$ $\delta_{3} m_{G}\left(\frac{p}{u^{0}}\right)^{\alpha}$.

Theorem 3 Suppose that (H3), (H4) and (H6) hold, $L<1$ and $\lambda=1$. Then

(i) (32) has a unique positive solution $u^{\star} \in C^{+}[0,1] \backslash\{\theta\}$ satisfying

$$
m_{u} g(t) \leq u^{\star}(t) \leq M_{u} g(t) \quad \text { for } t \in[0,1]
$$

where $0<m_{u}<M_{u}$ are constants.

(ii) For any $u_{0}(t) \in C^{+}[0,1] \backslash\{\theta\}$, the sequence

$$
\begin{aligned}
u_{n}(t) & =\left(Q u_{n-1}\right)(t)=\left(H F u_{n-1}\right)(t) \\
& =T F u_{n-1}+(T G) T F u_{n-1}+(T G)^{2} T F u_{n-1}+\cdots+(T G)^{n} T F u_{n-1}+\cdots
\end{aligned}
$$

$(n=1,2, \ldots)$ converges uniformly to the unique solution $u^{\star}$, and the rate of convergence is determined by

$$
\left\|u_{n}(t)-u^{\star}(t)\right\|=O\left(1-d^{\alpha^{n}}\right)
$$

where $0<d<1$ is a positive number.

Proof In view of (H3), (H4) and (H6), $Q: C^{+}[0,1] \rightarrow C^{+}[0,1]$ is a nondecreasing operator and satisfies $Q(\rho u) \geq \rho^{\alpha} Q(u)$ for $t \in[0,1]$ and $u \in C^{+}[0,1]$. Indeed, let $u_{\star}(t) \leq u_{\star \star}(t)$, $u_{\star}, u_{\star \star} \in C^{+}[0,1]$, since $f(s, u)$ is nondecreasing in $u$, then by using $f\left(s, u_{\star}(s)\right) \leq f\left(s, u_{\star \star}(s)\right)$, for $t \in[0,1]$, it follows that

$$
\begin{aligned}
T F u_{\star}(t) & =\int_{0}^{1} \int_{0}^{1} \int_{0}^{1} G_{1}(t, v) G_{2}(v, \tau) G_{3}(\tau, s) f\left(s, u_{\star}(s)\right) d s d \tau d v \\
& \leq \int_{0}^{1} \int_{0}^{1} \int_{0}^{1} G_{1}(t, v) G_{2}(v, \tau) G_{3}(\tau, s) f\left(s, u_{\star \star}(s)\right) d s d \tau d v=T F u_{\star \star}(t) .
\end{aligned}
$$

Moreover, from (25), it follows that for $T F u_{\star}(t) \leq T F u_{\star \star}(t)$

$$
G\left(T F u_{\star}\right)(t) \leq G\left(T F u_{\star \star}\right)(t) \quad \text { for } t \in[0,1]
$$

Finally, since $f(s, u)$ is nondecreasing in $u$, then by using form (34), $f\left(s, G\left(T F u_{\star}\right)(t)\right) \leq$ $f\left(s, G\left(T F u_{\star \star}\right)(t)\right)$, for $t \in[0,1]$, we have

$$
\begin{aligned}
(T G) T F\left(u_{\star}\right) & =\int_{0}^{1} \int_{0}^{1} \int_{0}^{1} G_{1}(t, v) G_{2}(v, \tau) G_{3}(\tau, s) f\left(s, G\left(T F u_{\star}\right)(s)\right) d s d \tau d v \\
& \leq \int_{0}^{1} \int_{0}^{1} \int_{0}^{1} G_{1}(t, v) G_{2}(v, \tau) G_{3}(\tau, s) f\left(s, G\left(T F u_{\star \star}\right)(s)\right) d s d \tau d v \\
& =(T G) T F u_{\star \star},
\end{aligned}
$$


i.e.,

$(T G) T F\left(u_{\star}\right) \leq(T G) T F u_{\star \star}$.

By induction, it is easy to see that

$$
(T G)^{n} T F\left(u_{\star}\right) \leq(T G)^{n} T F u_{\star \star}, \quad n=1,2, \ldots
$$

Hence, using (35), we have

$$
\begin{aligned}
Q\left(u_{\star}\right) & =T F\left(u_{\star}\right)+(T G) T F\left(u_{\star}\right)+(T G)^{2} T F\left(u_{\star}\right)+\cdots+(T G)^{n} T F\left(u_{\star}\right)+\cdots \\
& \leq T F\left(u_{\star \star}\right)+(T G) T F\left(u_{\star \star}\right)+(T G)^{2} T F\left(u_{\star \star}\right)+\cdots+(T G)^{n} T F\left(u_{\star \star}\right)+\cdots \\
& =Q\left(u_{\star \star}\right) .
\end{aligned}
$$

Now, we show that $Q: C^{+}[0,1] \rightarrow C^{+}[0,1]$ satisfies $Q(\rho u) \geq \rho^{\alpha} Q(u)$ for $t \in[0,1]$ and $u \in C^{+}[0,1]$. Note that

$$
\begin{aligned}
T F(\rho u) & =\int_{0}^{1} \int_{0}^{1} \int_{0}^{1} G_{1}(t, v) G_{2}(v, \tau) G_{3}(\tau, s) f(s, \rho u(s)) d s d \tau d v \\
& \geq \rho^{\alpha} \int_{0}^{1} \int_{0}^{1} \int_{0}^{1} G_{1}(t, v) G_{2}(v, \tau) G_{3}(\tau, s) f(s, u(s)) d s d \tau d v \\
& =\rho^{\alpha} T F(u) .
\end{aligned}
$$

Moreover, from (25), it follows that for $T F(\rho u) \geq \rho^{\alpha} T F(u)$,

$$
\begin{aligned}
G(T F \rho u)(t) & \geq G\left(\rho^{\alpha} T F(u)\right)(t) \\
& =\rho^{\alpha} G(T F(u))(t) \quad \text { for } t \in[0,1] .
\end{aligned}
$$

Finally, we have

$$
\begin{aligned}
(T G) T F(\rho u)(t) & =\int_{0}^{1} \int_{0}^{1} \int_{0}^{1} G_{1}(t, v) G_{2}(v, \tau) G_{3}(\tau, s) f(s, G(T F \rho u)(s)) d s d \tau d v \\
& \geq \int_{0}^{1} \int_{0}^{1} \int_{0}^{1} G_{1}(t, v) G_{2}(v, \tau) G_{3}(\tau, s) f\left(s, \rho^{\alpha} G(T F(u))(s)\right) d s d \tau d v \\
& \geq \rho^{\alpha^{2}} \int_{0}^{1} \int_{0}^{1} \int_{0}^{1} G_{1}(t, v) G_{2}(v, \tau) G_{3}(\tau, s) f(s, G(T F(u))(s)) d s d \tau d v \\
& =\rho^{\alpha^{2}}(T G) T F(u)(t),
\end{aligned}
$$

i.e.,

$$
(T G)(T F \rho u)(t) \geq \rho^{\alpha^{2}}(T G) T F(u)(t) .
$$

By induction, it is easy to see that

$$
(T G)^{n}(T F \rho u)(t) \geq \rho^{\alpha^{n+1}}(T G) T F(\rho u)(t), \quad n=1,2, \ldots .
$$


Hence, using (35) and $\rho \in(0,1), \alpha \in(0,1)$, we have

$$
\begin{aligned}
Q(\rho u) & =T F(\rho u)+(T G) T F(\rho u)+(T G)^{2} T F(\rho u)+\cdots+(T G)^{n} T F(\rho u)+\cdots \\
& \geq \rho^{\alpha} T F(u)+\rho^{\alpha^{2}}(T G) T F(u)+\rho^{\alpha^{3}}(T G)^{2} T F(u)+\cdots+\rho^{\alpha^{n+1}}(T G)^{n} T F(u)+\cdots \\
& \geq \rho^{\alpha} T F(u)+\rho^{\alpha}(T G) T F(u)+\rho^{\alpha}(T G)^{2} T F(u)+\cdots+\rho^{\alpha}(T G)^{n} T F(u)+\cdots \\
& =\rho^{\alpha}\left(T F(u)+(T G) T F(u)+(T G)^{2} T F(u)+\cdots+(T G)^{n} T F(u)+\cdots\right) \\
& =\rho^{\alpha} Q(u) .
\end{aligned}
$$

By Lemma 13, there exists $0<s_{g} \leq S_{g}$ such that

$$
s_{u} g(t) \leq Q g(t) \leq S_{u} g(t) .
$$

Let

$$
s=\sup \left\{s_{g}: s_{u} g(t) \leq Q g(t)\right\}, \quad S=\inf \left\{S_{g}: Q g(t) \leq S_{u} g(t)\right\} .
$$

Pick $m_{s}$ and $M_{s}$ such that

$$
0<m_{s}<\min \left\{1, s^{\frac{1}{1-\alpha}}\right\}
$$

and

$$
\max \left\{1, S^{\frac{1}{1-\alpha}}\right\}=M_{s}<\infty
$$

Set $u_{0}(t)=m_{s} g(t), v_{0}(t)=M_{s} g(t), u_{n}=Q u_{n-1}$, and $v_{n}=Q v_{n-1}, n=1,2, \ldots$ From (36) and (38), we have

$$
m_{s} g(t)=u_{0}(t) \leq u_{1}(t) \leq \cdots \leq u_{n}(t) \leq \cdots \leq v_{n}(t) \leq \cdots \leq v_{1}(t) \leq v_{0}(t)=M_{s} g(t)
$$

Indeed, from (39) $m_{s}<1$, and $m_{s}^{\alpha-1} s>1$, we have

$$
\begin{aligned}
u_{1}(t) & =Q\left(u_{0}\right)=Q\left(m_{s} g(t)\right) \geq m_{s}^{\alpha} Q(g(t)) \geq m_{s}^{\alpha} s g(t) \\
& =m_{s}^{\alpha-1} s m_{s} g(t)=m_{s}^{\alpha-1} s u_{0}(t) \geq u_{0}(t),
\end{aligned}
$$

and by induction

$$
u_{n+1}(t)=Q\left(u_{n}\right) \geq Q\left(u_{n-1}\right)=u_{n}(t) .
$$

From (40), $M_{s}>1$, and $M_{s}^{\alpha-1} S<1$, we have

$$
\begin{aligned}
v_{1}(t) & =Q\left(v_{0}\right) \leq M_{s}^{\alpha} Q(g(t))=M_{s}^{\alpha} Q\left(\frac{1}{M_{s}} v_{0}\right)=M_{s}^{\alpha} Q(g) \\
& \leq M_{s}^{\alpha} S g \leq S M_{s}^{\alpha-1} M_{s} g=S M_{s}^{\alpha-1} v_{0}(t) \leq v_{0}(t),
\end{aligned}
$$


and by induction

$$
v_{n+1}(t)=Q\left(v_{n}\right) \leq Q\left(v_{n-1}\right)=v_{n}(t) .
$$

Let $d=\frac{m_{s}}{M_{s}}$. Then

$$
u_{n} \geq d^{\alpha^{n}} v_{n}
$$

In fact $u_{0}=d v_{0}$ is clear. Assume that (42) holds with $n=k$ ( $k$ is a positive integer), i.e., $u_{k} \geq d^{\alpha^{k}} v_{k}$. Then

$$
u_{k+1}=Q\left(u_{k}\right) \geq Q\left(d^{\alpha^{k}} v_{k}\right) \geq\left(d^{\alpha^{k}}\right)^{\alpha} Q\left(v_{k}\right)=d^{\alpha^{k+1}} Q\left(v_{k}\right)=d^{\alpha^{k+1}} v_{k+1} .
$$

By induction, it is easy to see that (42) holds. Furthermore, in view of (38), (41) and (42), we have

$$
0 \leq u_{n+z}-u_{n} \leq v_{n}-u_{n} \leq\left(1-d^{\alpha^{n}}\right) v_{0}=\left(1-d^{\alpha^{n}}\right) M_{s} g(t)
$$

and

$$
\left\|u_{n+z}-u_{n}\right\| \leq\left\|v_{n}-u_{n}\right\| \leq\left(1-d^{\alpha^{n}}\right) M_{s}\|g\|
$$

where $z$ is a nonnegative integer. Thus, there exists $u^{\star} \in C^{+}[0,1]$ such that

$$
\lim _{n \rightarrow \infty} u_{n}(t)=\lim _{n \rightarrow \infty} v_{n}(t)=u^{\star}(t) \quad \text { for } t \in[0,1]
$$

and $u^{\star}(t)$ is a fixed point of $Q$ and satisfies

$$
m_{g} g(t) \leq u^{\star}(t) \leq M_{g} g(t)
$$

This means that $u^{\star} \in C_{\star}^{+}[0,1]$, where $C_{\star}^{+}[0,1]=\left\{u \in C^{+}[0,1], u(t)>0\right.$ for $\left.t \in(0,1)\right\}$.

Next we show that $u^{\star}$ is the unique fixed point of $Q$ in $C_{\star}^{+}[0,1]$. Suppose, to the contrary, that there exists another $\bar{u} \in C_{\star}^{+}[0,1]$ such that $Q \bar{u}=\bar{u}$. We can suppose that

$$
u^{\star}(t) \leq \bar{u}(t), \quad u^{\star}(t) \neq \bar{u}(t) \quad \text { for } t \in[0,1] .
$$

Let $\widehat{\tau}=\sup \left\{0<\tau<1: \tau u^{\star} \leq \bar{u} \leq \tau^{-1} u^{\star}\right\}$. Then $0<\widehat{\tau} \leq 1$ and $\widehat{\tau} u^{\star} \leq \bar{u} \leq \widehat{\tau}^{-1} u^{\star}$. We assert $\widehat{\tau}=1$. Otherwise, $0<\widehat{\tau}<1$, and then

$$
\begin{aligned}
& \bar{u}=Q \bar{u} \geq Q\left(\widehat{\tau} u^{\star}\right) \geq \widehat{\tau}^{\alpha} Q\left(u^{\star}\right)=\widehat{\tau}^{\alpha} u^{\star}, \\
& u^{\star}=Q u^{\star} \geq Q(\widehat{\tau} \bar{u}) \geq \widehat{\tau}^{\alpha} Q(\bar{u})=\widehat{\tau}^{\alpha} \bar{u} .
\end{aligned}
$$

This means that $\widehat{\tau}^{\alpha} u^{\star} \leq \bar{u} \leq\left(\widehat{\tau}^{\alpha}\right)^{-1} u^{\star}$, which is a contradiction of the definition of $\widehat{\tau}$, because $\widehat{\tau}<\widehat{\tau}^{\alpha}$. 
Let us introduce the following notations for $\mu=0$

$$
\begin{gathered}
T_{\lambda} u(t):=T F u(t)=\lambda \int_{0}^{1} \int_{0}^{1} \int_{0}^{1} G_{1}(t, v) G_{2}(v, s) G_{3}(s, \tau) f(\tau, u(\tau)) d \tau d s d v, \\
Q_{\lambda} u:=H F u=T F u+(T G) T F u+(T G)^{2} T F u+\cdots+(T G)^{n} T F u+\cdots \\
=T_{\lambda} u+(T G) T_{\lambda} u+(T G)^{2} T_{\lambda} u+\cdots+(T G)^{n} T_{\lambda} u+\cdots,
\end{gathered}
$$

i.e., $Q_{\lambda} u=\lambda Q u$, where $Q$ is given by (33).

Theorem 4 Suppose that (H3), (H4), (H6) and $L<1$ hold. Then (32) has a unique positive solution $u_{\lambda}(t)$ for any $0<\lambda \leq 1$.

Proof Theorem 3 implies that for $\lambda=1$, the operator $Q_{\lambda}$ has a unique fixed point $u_{1} \in$ $C^{+}[0,1]$, that is $Q_{1} u_{1}=u_{1}$. Then from Lemma 10 , for every $\lambda_{\star} \in(0,1)$, there exists a function $u_{\star} \in P \backslash\{\theta\}$ such that $Q_{\lambda_{\star}} u_{\star}=u_{\star}$.

Thus, $u_{\lambda}$ is a unique positive solution of (32) for every $0<\lambda \leq 1$.

\section{Application}

As an application of Theorem 1, consider the sixth-order boundary value problem

$$
\begin{aligned}
& -u^{(6)}+\left(1-0.5 t^{2}\right) u^{(4)}+(4.5-0.5 \sin \pi t) u^{\prime \prime}+C(-5+\cos 0.5 \pi t) u \\
& =(0.5 t(1-t)+u) \varphi+\lambda\left(1+\sin \pi t+u^{2}\right), \quad 0<t<1, \\
& -\varphi^{\prime \prime}+2 \varphi=\mu u, \quad 0<t<1, \\
& u(0)=u(1)=u^{\prime \prime}(0)=u^{\prime \prime}(1)=u^{(4)}(0)=u^{(4)}(1)=0, \\
& \varphi(0)=\varphi(1)=0,
\end{aligned}
$$

for a fixed $\lambda_{1}=2, \lambda_{2}=-2, \lambda_{3}=1$ and $\varkappa=2$. In this case, $a=\lambda_{1}+\lambda_{2}+\lambda_{3}=1, b=-\lambda_{1} \lambda_{2}-$ $\lambda_{2} \lambda_{3}-\lambda_{1} \lambda_{3}=4$, and $c=\lambda_{1} \lambda_{2} \lambda_{3}=-4$. We have $A(t)=1-0.5 t^{2}, B(t)=4.5-0.5 \sin \pi t$, $C(t)=-5+\cos 0.5 \pi t, D(t)=0.5 t(1-t)$ and $f(t, u)=1+\sin \pi t+u^{2}$. It is easy to see that $\pi^{6}+$ $a \pi^{4}-b \pi^{2}+c=1,015.3>0, a=\sup _{t \in[0,1]} A(t), b=\inf _{t \in[0,1]} B(t)$ and $c=\sup _{t \in[0,1]} C(t)$. Note also that $K=\max _{0 \leq t \leq 1}[-A(t)+B(t)-C(t)-(-a+b-c)]=2, D_{2}=\max _{t \in[0,1]} \int_{0}^{1} G_{2}(t, v) d v=$ 0.15768, $C=\max _{t \in[0,1]} D(t)=0.125, d_{1}=\max _{t \in[0,1]} \int_{0}^{1} G(t, s) d s=0.10336, \mu^{* *}=\frac{1-D_{2} K}{D_{2} C d_{1}}=$ 336.1 and $D_{2} K=0.3153<1$. Thus, if $0<\mu<336.1$, then the conditions of Theorem 1 (note $\left.L=D_{2}\left(K+\mu C d_{1}\right)<1\right)$ are fulfilled (in particular, (H3)-(H5) are satisfied). As a result, Theorem 1 can be applied to (43).

\section{Competing interests}

The authors did not provide this information.

\section{Authors' contributions}

The authors did not provide this information.

\section{Author details}

'Department of Mathematics, Texas A\&M University-Kingsville, 700 University Blvd., Kingsville, 78363-8202, USA. ${ }^{2}$ Department of Analysis, University of Miskolc, Egyetemvaros, 3515, Hungary. ${ }^{3}$ School of Mathematics, Statistics and Applied Mathematics, National University of Ireland, Galway, Ireland. 


\section{References}

1. Evans, JD, Galactionov, VA, King, JR: Unstable sixth-order thin film equation: II. Global similarity patterns. Nonlinearity 20, 1843-1881 (2007)

2. Yude, J, Yanping, G, Yukun, Y, Yingjie, F: Nodal solutions for sixth-order $m$-point boundary-value problems using bifurcation methods. Electron. J. Differ. Equ. 2012, Article ID 217 (2012)

3. Liu, C, Liu, A, Tang, H: Time-periodic solutions for a driven sixth-order Cahn-Hilliard type equation. Bound. Value Probl. 2013, Article ID 73 (2013)

4. Alvelid, M: Sixth order differential equation for sandwich beam deflection including transverse shear. Compos. Struct. 102, 29-37 (2013)

5. Liu, X, Li, W: Positive solutions for the nonlinear fourth-order beam equation with three parameters. J. Math. Anal. Appl. 303, 150-163 (2005)

6. Li, W: The existence and multiplicity of positive solutions of nonlinear sixth-order boundary value problem with three variable coefficients. Bound. Value Probl. 2012, Article ID 22 (2012)

7. El-Gebeily, MA, O'Regan, D, Nieto, JJ: A monotone iterative technique for stationary and time dependent problems in Banach apaces. J. Comput. Appl. Math. 233, 2395-2404 (2010)

8. Agarwal, RP, Kovacs, B, O'Regan, D: Existence of positive solution for a sixth-order differential system with variable parameters. J. Appl. Math. Comput. (2013). doi:10.1007/s12190-013-0701-1

9. Nieto, JJ: Generalized quasilinearization method for a second order ordinary differential equation with Dirichlet boundary conditions. Proc. Am. Math. Soc. 125, 2599-2604 (1997)

10. Guo, D, Lakshmikantham, V: Nonlinear Problems in Abstract Cones. Academic Press, New York (1988)

11. Chai, G: Existence of positive solutions for fourth-order boundary value problem with variable parameters. Nonlinear Anal. 66, 870-880 (2007)

doi:10.1186/1687-2770-2013-184

Cite this article as: Agarwal et al.: Positive solutions for a sixth-order boundary value problem with four parameters.

Boundary Value Problems 2013 2013:184.

\section{Submit your manuscript to a SpringerOpen ${ }^{\ominus}$ journal and benefit from:}

- Convenient online submission

- Rigorous peer review

- Immediate publication on acceptance

Open access: articles freely available online

High visibility within the field

- Retaining the copyright to your article 